\title{
Effect of Illuminant on Colour Perception Concerning the Simulation of Colour Deficiencies
}

This paper was downloaded from TechRxiv (https://www.techrxiv.org).

LICENSE

CC BY-SA 4.0

SUBMISSION DATE / POSTED DATE

04-02-2022 / 11-02-2022

CITATION

Zajaczkowski, Maciej (2022): Effect of Illuminant on Colour Perception Concerning the Simulation of Colour Deficiencies. TechRxiv. Preprint. https://doi.org/10.36227/techrxiv.19123046.v1

DOI

10.36227/techrxiv.19123046.v1 


\title{
Effect of Illuminant on Colour Perception Concerning the Simulation of Colour Deficiencies
}

\author{
Maciej ZajaczKowsKi
}

February 4, 2022

\begin{abstract}
Normal human colour vision relies on 3 different cone photoreceptors ( $L, M$, and $S)$ in the retina and 2 opponent processes: red-green and blue-yellow. However, the exact hue perceived when observing an object is dependent upon three main factors, namely the genes encoding the opsins present in the observer's eye, the spectral reflectivity of the object as well as the spectral composition of the incident light, also known as the illuminant. By controlling the composition of the illuminant, the amount of available light that can be reflected by an object can be controlled, which in turn affects the size of the observer's colour gamut. By choosing illuminant spectra mostly/only containing colours that are most prominent to people affected by colour deficiencies, we can replicate the reduced colour gamut that they observe. Conversely, this technique can also be used to enhance certain colours and equalise the brightness of hues across the spectrum.
\end{abstract}

\section{INTRODUCTION}

Typical human colour vision is trichromatic meaning that three primaries are necessary to recreate all visible colours and can be thought of as three dimensional, as demonstrated by the RGB colour space. This is brought about by the activation of long $(\mathrm{L})$, medium $(\mathrm{M})$, and short (S) cones which roughly correspond to red, green, and blue. This is not the case for everyone, with about $1 / 12$ men and 1/200 women having some form of colour deficiency; the sex bias being caused by $\mathrm{X}$-linked recessive inheritance of red-green deficiencies. These are far more common because red-green vision is quite a recent development in the evolution of colour vision in primates. [1][2]

Of these red-green deficiencies, the majority are partial and occur when either the "'red cone" detects too much green light (protanomaly), and the "green cone" detects too much red light (deuteranomaly). The result is a decreased colour gamut, however, it can sometimes be quite mild and only specific shades are affected. In this case, the affected person simply sees some colours in less contrast, will probably fail colorblind tests but rarely confuses colours in everyday life. In more severe cases, the affected person will confuse some colours such as reds with browns, blues with purples, or greens and oranges.

Dichromacies occur when a person lacks a gene for producing one type of photopigment and only has two types of functioning cones. This means that their colour vision is reduced to two dimensions and they only require two primaries to recreate any colour that they can perceive.

People affected by protanopia and deuteranopia will only see the world in yellows/yellowish-greens, blues, and browns. As such red, orange, yellow and green (every colour from red to cyan) and will be a shade of yellow and blue, and purple (every colour from cyan to magenta) will be a shade of blue. Cyan on the other hand will be perceived as grey as it lies on the neutral point in protanopes and deuteranopes and thus causes equal activation of two cone cells. [3][4]

Relating more to this paper, the colour gamut can also be restricted by limiting the spectrum of the illuminant light; for example, if no red light is shone on a red object, it will no longer look red. A similar effect is seen with the low pressure sodium lamp which emits yellow light at $589 \mathrm{~nm}$.[7] As such, the spectrum of the illuminant can be carefully adjusted to only contain wavelengths corresponding to colours most visible to colours deficient people. Figure 1 shows the rough limited spectrum to colour deficiency phenotype equivalence, where stimulating normal vision with a limited range of colours (left images) simulates a scenario similar to that of a colour deficient person with fewer types of functional cone cells in their eyes.[5][6] 
This type of simulation, however, does have a number of limitations, primarily the difference in brightness of specific colours. For example, trying to simulate protanopia with only green and blue light will lead to red being almost black. However, in the case of a protanope, their green $(\mathrm{M})$ cone still detects some red light leading to the perception of a dark brown colour instead of black. [7]

In addition, everyone will experience a change in colours when viewing objects under the simulation illuminant. As illustrated by figure 2, colour deficient people will still experience a change in colours albeit a smaller intensitybased change, without an alteration of the dimensions of their colour space for non-partial (-anopia) types of colour deficiency. This has been confirmed with subjective experiences of strongly protanopic subjects.

\section{Methods}

A 12 channel (10 excluding far-red and UV) device was built in order to precisely replicate any spectrum through fine control over each part of the spectrum ranging from far-red, deep red, red, orange, yellow, chartreuse, green, cyan, blue, royal blue, violet and ultraviolet. [8] For each experiment, the device was set up in a dark room with colour vision deficiency (CVD) simulation spectra were then determined based on the colours that dominate colour deficient vision. Simulations were then evaluated using colour charts and Ishihara plates, and subjects with normal and deficient vision were asked to describe their personal observations to help fine tune and confirm simulations.

Summary of choices of light spectra for the simulation of each type of colour deficiency:

- To simulate rod monochromacy, only pure cyan light was used. This was chosen based on the peak sensitivity of the rod cells and is sufficient to stimulate only one type of cell.

- For protanopia blue and green lights where used as this will only stimulate the $\mathrm{M}$ and $\mathrm{S}$ cones in the subjects retina, emulating the phenotype of a protanope with only $\mathrm{M}$ and $\mathrm{S}$ cones present in their retina.

- For Deuteranopia - pure yellow and blue lights are chosen, simulating a situation where a person only has red (L) and blue (S) cones

- For Tritanopia: a condition where only red and green cones are present, can be simulated with pure red and cyan lights.

- The colour spectra for anomalous trichromacies
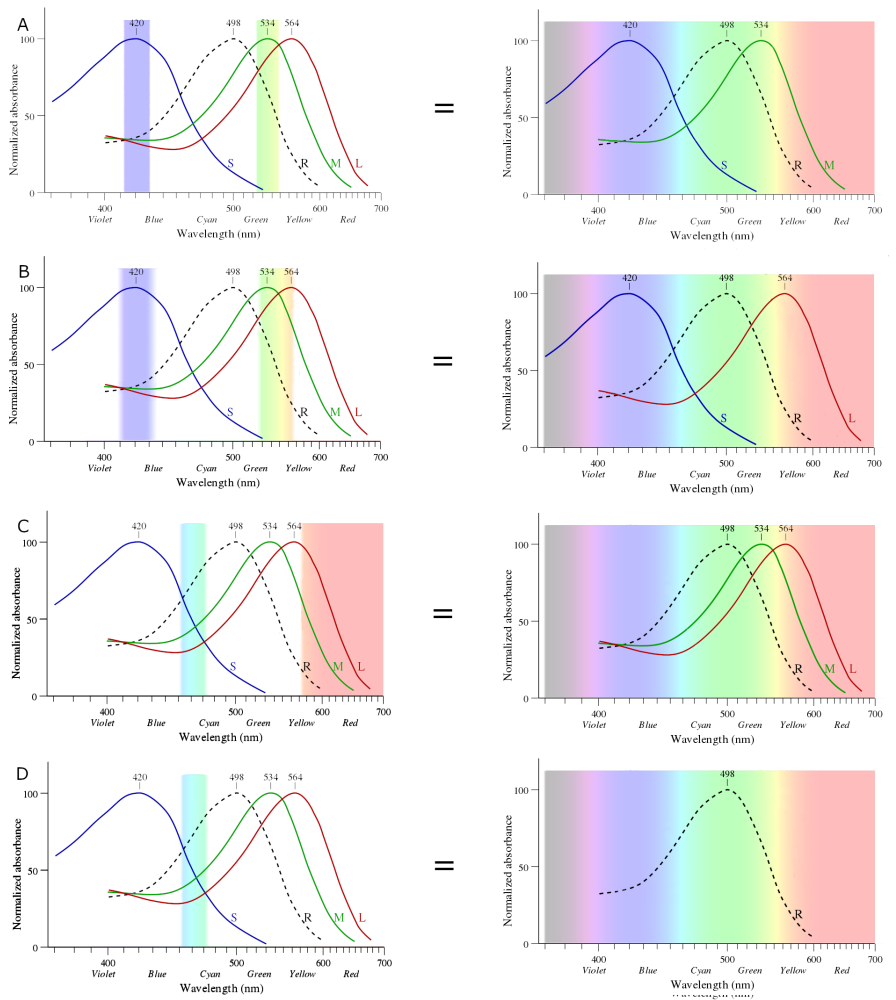

Figure 1: Diagram showing overlay of spectrum and cone cell responsivities to portray that the effective result of viewing objects under a restricted light source (left) is equivalent to viewing under a full spectrum with a colour deficient cone cell phenotype as show on the right A protanopia - using only blue and green light (cyan can also be added) simulates a missing $L$ cone $\boldsymbol{B}$ - using blue and yellow light simulates a missing $M$ cone - deuteranopia $C$ red and cyan light simulate tritanopia $\boldsymbol{D}$ cyan light simulates colours viewed with rod monochromacy 

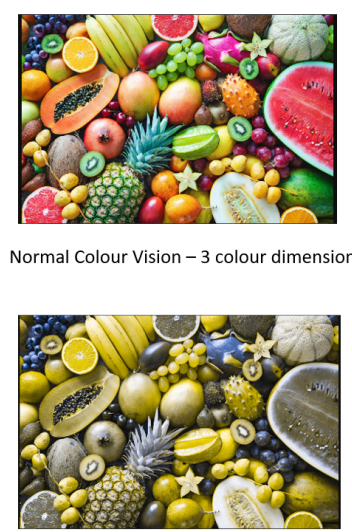

Protanopia Vision -2 colour dimensions
Normal Colour Vision - 3 colour dimensions

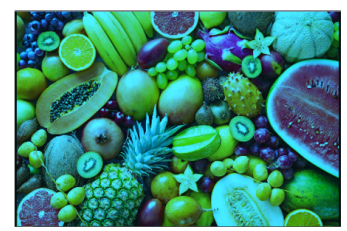

Transformed to 2 colour dimensions with lamp

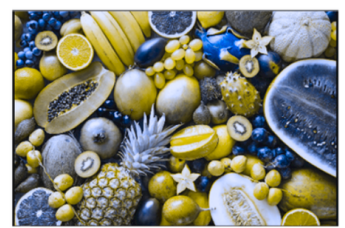

Transformed to different 2 colour dimensions
Figure 2: Demonstration of possible manner in which limited light source affects the view of viewers with normal (top images) and colour deficient vision (bottom images). The figure demonstrates that people with colour deficiencies will still experience a change, however, it will not be as drastic and the dimensions of their colour space will not change as in the case of complete deficiencies of each cone type: protanopia, deuteranopia and tritanopia. People with colour anomalies will still observed a decrease in colour space dimension under 'full' simulations.

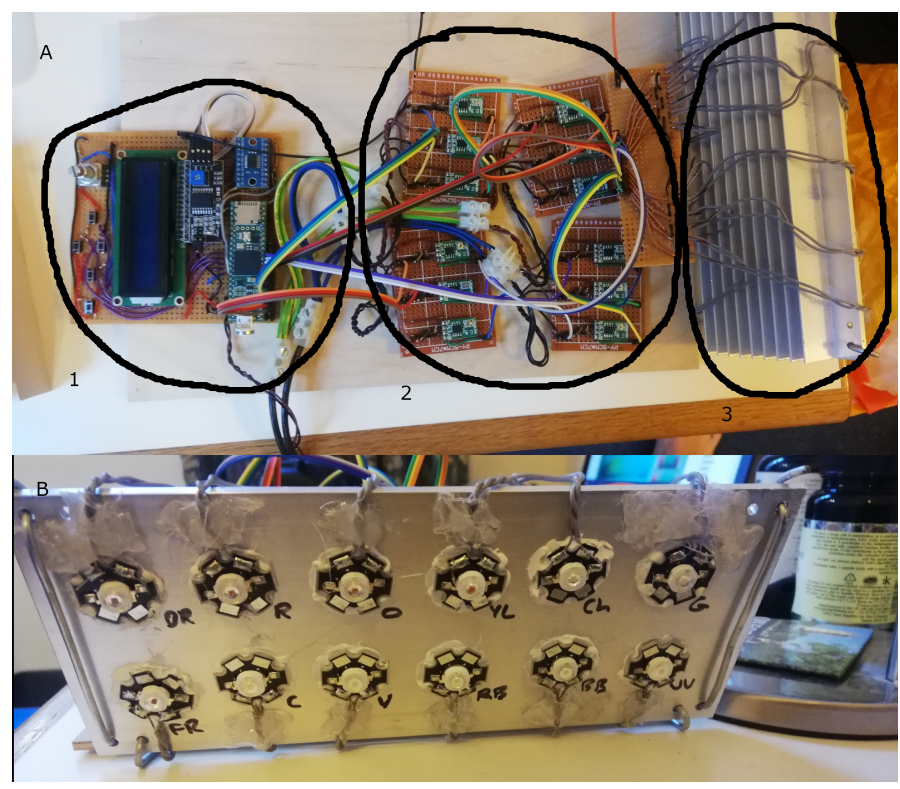

Figure 3: $A$ - bird eye view of apparatus containing; 1 - control board, 2-current sources and 3 - lights $\boldsymbol{B}$ - front view of lights with each colours labeled (see letters) where more complicated and were created by adding amounts of red and green to the simulations for protanopia and deuteranopia.

Anomalous Trichromacies are more difficult to simulate but varying the light intensity (i.e. small amount of red) or using wavelengths closer to yellow, such as orange, chartreuse/green, and blue LEDs should be able to simulate mild-moderate red-green colour deficiencies. For tritanomaly, try cyan, green and red.

\section{Results}

Figures 4 and 5 show the effects of the lamp with different spectra on the perceived colours in a colour spectrum sheet and an Ishihara plate - the original test sheet/plate is portrayed in subfigure N1. This represents normal vision with a 'full' spectrum of colours being visible in figure 4 and the numbers 26 and 42 being equally obvious to a person with typical colour vision.

Subfigure M1 represents the simulation of colour intensity rod monochromacy. In both Figures 4 and 5, almost no colour information is visible and the cyan-blue region of the spectrum is the brightest, which is expected as this is the region of peak rod responsivity and thus represents a successful simulation. For the Ishihara plate, the numbers are invisible with the exception of the 2 in the left plate however, this is only caused due to an intensity difference instead of colour.

For figure 4 - the series P1, D1, and T1 represent the three main non-partial colour deficiencies: protan, deutan and tritanopia and the series P2, D2 and T2 below represents the processing of subfigure N1 to simulate the three colours deficiencies. By comparing series 1 and 2, we can see that the images are indeed strikingly similar meaning that the simulation has been successful.

Figure 5 has a similar layout to figure 4 , row 1 shows the illuminant-based simulation and row 2 shows the computer-based simulation. For P1 and P2, the number on the right-hand side of each plate (6 and 2) is more obvious and saturated for both types of simulation, which is the expected result for protanopia. For D1 and D2, the opposite is true; the numbers on the left-hand side of each plate (2 and 4) are more visible and colourful for both simulations; again the expected result for deuteranopia. Finally, for T1 and $\mathrm{T} 2$, both numbers are clearly visible but are redder than the ones in the original image which again indicates a successful simulation. 


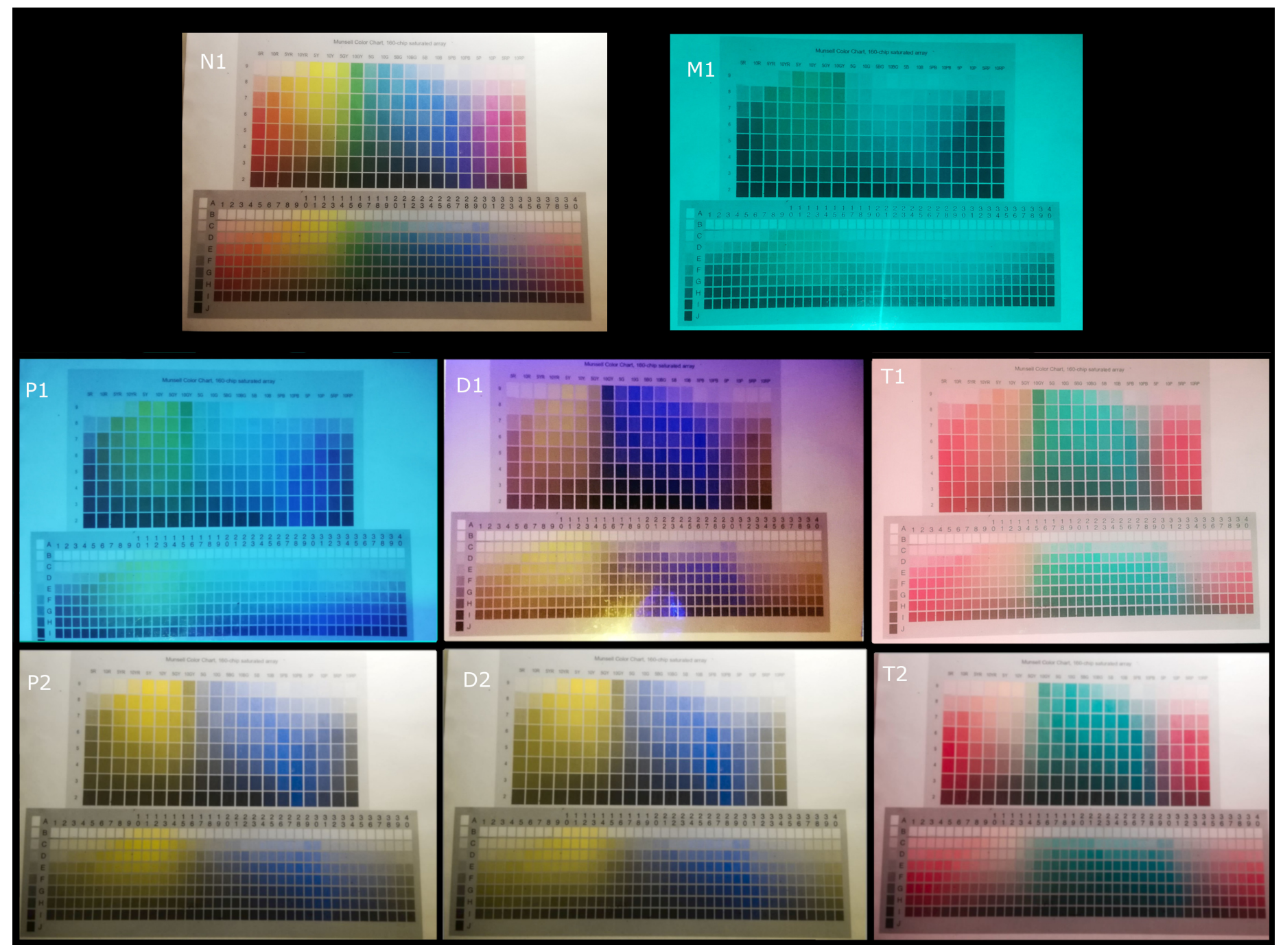

Figure 4: N1 - original image of colour spectrum sheet. M1 - monochrome simulation using only cyan light - colours are almost non-existent and the cyan-blue region is the brightest P1, D1 and T1- simulated colour deficiencies using lamp P2, D2, and T2 N1 test sheet with colour deficiencies simulated using image processing algorithm for comparison (from https://www.color-blindness.com/coblis-color-blindnesssimulator/) 


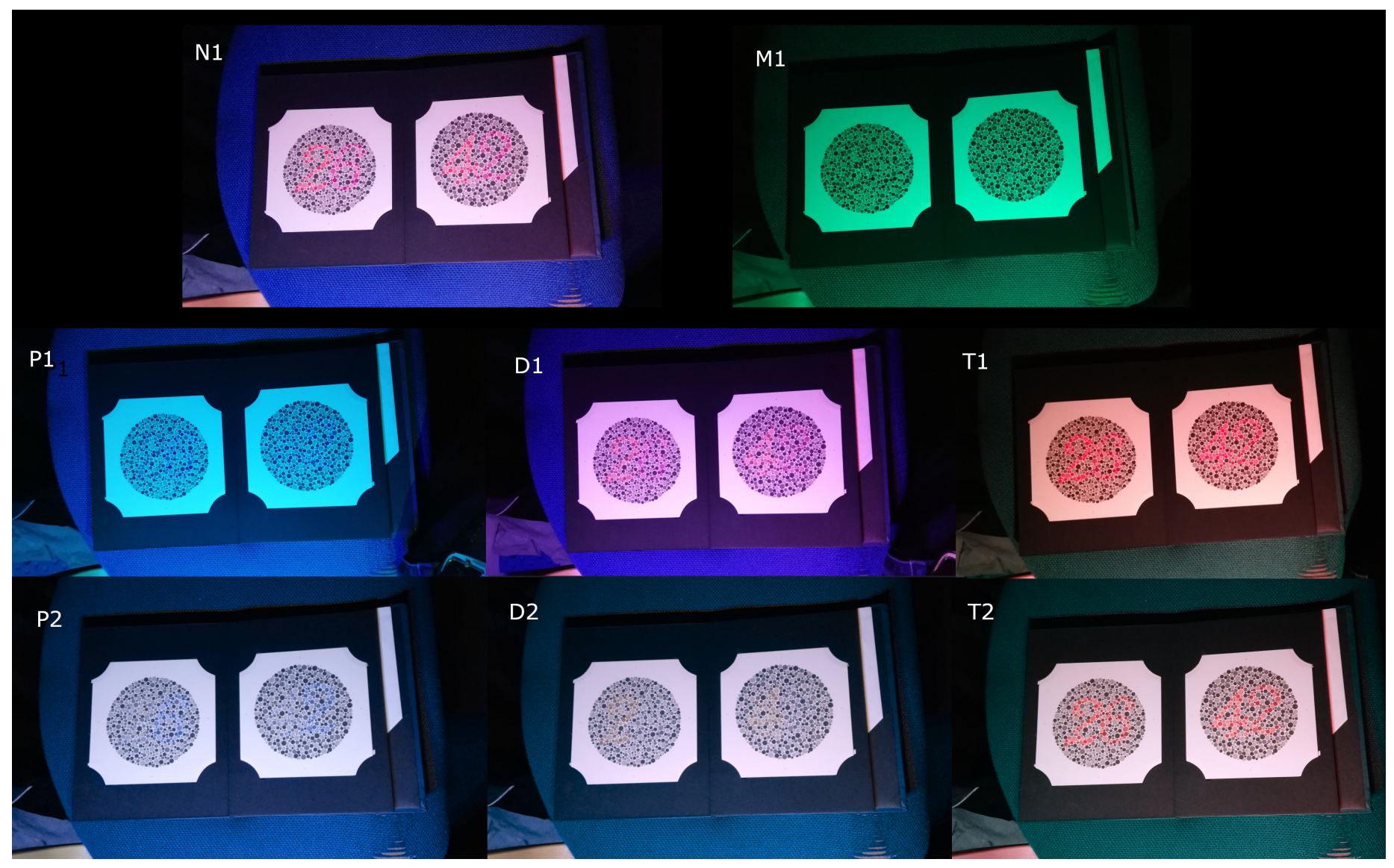

Figure 5: N1 - original image of colour spectrum sheet. M1 - monochrome simulation using only cyan light - numbers are mostly unclear P1, D1 and T1 - simulated colour deficiencies using lamp P2, D2, and T2 N1 test sheet with colour deficiencies simulated using image processing algorithm for comparison (from https://www.color-blindness.com/coblis-color-blindness-simulator/) P3, D3, and T3 same as P2, D2, and T2 but with white balance correction 


\section{Discussion}

Comparing illuminant-based and computed simulations revealed that they are quite similar. Combined with personal observation of visible colours and involvement of several test subjects including colour deficient viewers, we can say that the simulations are surprisingly accurate at giving a first-hand experience of colour deficiencies.

There are however limitations to this method of simulation; these relate to the excessive darkening of some colors due to a limited spectrum light source as well as imperfections in the led lights. The former relates to how not illuminating red objects with any red light will make them appear very dark, however, this is not the case for protanopes as their remaining $\mathrm{M}$ cone still detects some red light. The same is true for green when only yellow light is present in some cases. The latter relates to the fact that the emission spectrum of LED lights is a bell-curve, thus yellow light will emit a bit of orange and green, skewing the simulations. This small amount of unwanted red light is visible in figure 4 subfigure D1 where the red and magenta areas of the spectrum are not shades of greyish brown, rather desaturated shades of pinkish red.

\section{Conclusion}

I have managed to demonstrate that tuning the spectrum of the illuminant in a precise manner can indeed have a highly tunable effect on the perception of colours and can also create accurate simulations of common colour vision deficiencies. Additionally, using lights instead of optical filters makes this a cheaper and more customisable solution with the only limitation of requiring a dark environment. Furthermore, this technology could be used for applications where it would be useful to modify specific to make them more or less visible, such as examining the quality of food and other product, spotting counterfeit money, and masking colours to reveal hidden nuances.

\section{AcKNowledgments}

I would like to thank all of the subjects who participated in subjective evaluation of their perception of colours under the lamp, especially those with colour deficiencies.

\section{REFERENCES}

[1] Neitz, J., Neitz, M. (2011). The genetics of normal and defective color vision, Vision Research, https://doi.org/10.1016/J.VISRES.2010.12.002

[2] Carvalho, L. S. et al., (2017). The genetic and evolutionary drives behind primate color vision, Frontiers in Ecology and Evolution, https://doi.org/10.3389/FEVO.2017.00034/BIBTEX

[3] Colourblindessawareness (2022) accessed from: https:/ /www.colourblindawareness.org/

[4] Kalloniatis, M., Luu, C. (2007), The Perception of Color. Webvision: The Organization of the Retina and Visual System https://www.ncbi.nlm.nih.gov/books/NBK11538/

[5] Singh B et al., (2009), Radiosterilization of Fluoroquinolones and Cephalosporins: Assessment of Radiation Damage on Antibiotics by Changes in Optical Property and Colorimetric Parameters, https:/ / www.researchgate.net/ figure/The-colortriangle-interaction-of-illuminant-source-a-object-sreflectance_fig1_23789543

[6]Lotto, R. B., Purves, D. (2000). An empirical explanation of color contrast, Proceedings of the National Academy of Sciences, https://doi.org/10.1073/PNAS.210369597

[7] Sodium | Elements | RSC Education, (n.d.). from: https://edu.rsc.org/elements/sodium/2000032.article

[8] 1W / 3W LED with PCB (2022), Bridgelux and EPILED LEDs, accessed from: https:/ / futureeden.co.uk/ 